\title{
ULTRA SONIC RANGE FINDING FOR DISTANCE MEASURING IN COAL MINING
}

\author{
Sneha Punia ${ }^{1}$, Ramandeep Singh ${ }^{2}$ \\ ${ }^{1}$ M.Tech (E.S), ITM University, Gurgaon \\ ${ }^{2}$ Assistant Professor, ITM University, Gurgaon
}

\begin{abstract}
Measuring distances has always been a challenge in coal mines as it involves a lot of risks and disturbances. One method to measure the distances without any hindrances in sensitive places like coal mines is via Ultra Sonic waves which generates $40 \mathrm{KHz}$ sound pulse that travels with the speed of sound to the object and returns it echo that helps in calculating the distance, which further display on the LCD display. The medium required here is air medium. This project performs the distance measuring techniques, required in coal mines. This technique is proven to be very helpful in places where human reach is not possible or difficult.
\end{abstract}

Keywords: UltraSonic Sensors, Range Finding, AVR Microcontroller.

$* * *$

\section{INTRODUCTION}

Ultra sound is a popular technique of sensing distance of objects. It is fast, accurate and do not create disturbances in human activities. The main idea of this paper is to find out the range of applications and to learn the possibilities and advantages of an Ultra Sonic sensor in the measuring world. In this paper we will learn to measure the distances by sending a $40 \mathrm{KHz}$ sound wave and get back the echo for calculating time and distance. Microcontroller ATmega128 is used to generate the pulses, calculating the distance by sensors and displaying on a LCD display. Before Ultra Sonic waves the other method uses many a number of sensors and then detects the distance. Ultra Sonic range finder uses less number of detectors and measures the distance to the surface of the required object.

If the transmitter does not find the suitable surface the LCD displays an error indicating distance out of range or fails to calculate the distance.

\section{BLOCK DIAGRAM}

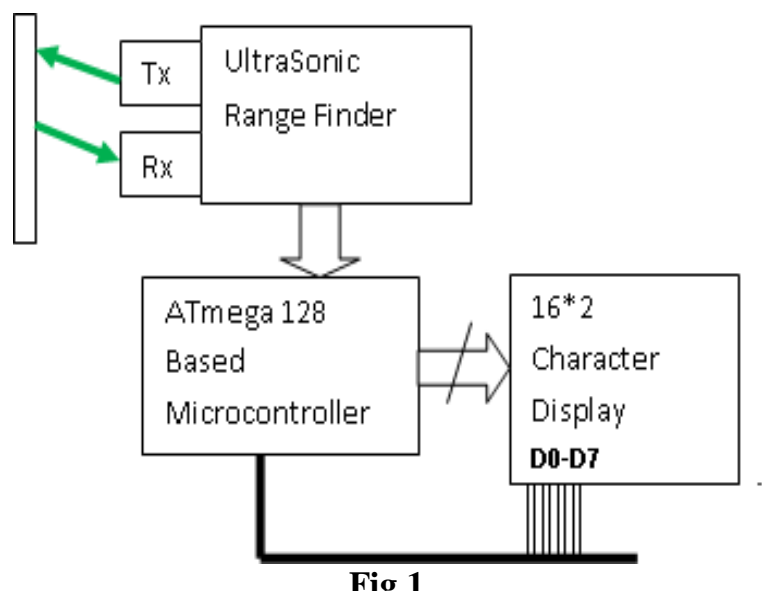

Fig 1
The distance of an object is measured by sending Ultra Sonic waves from a transmitter towards the object, the waves colliding with the object returns an echo to the sensor. The time taken by the waves is calculated by the sensor and sent to the microcontroller which controls the measurement and sends it to the LCD display showing the measured distance in centimeters.

\section{ALGORITHM}

When program starts, a pulse of $10 \mathrm{~ms}$ is triggered towards the sensor. Mostly these pulses get easily distorted by the narrow band response of the transducer.[1][2] To avoid such distortions small cycles up to 15 cycles are used for the transducer excitement. Once pulse triggers echo is produced by the transducer. The sensor produces echo in a similar way bats produces the ultrasonic noise. Status of echo is monitored at regular intervals of time and high status at echo pin is checked. When the timer gets started echo error is checked. After checking echo error the distance is calculated based on the pulse width of echo signal. Due to temperature variations $\pm 0.2 \% /{ }^{\circ} \mathrm{C}$ error can be observed and considered into the calculations. As during mining the temperature increases/decreases rapidly when distance increases and decreases. The distance measured in centimeters is calculated by dividing the elapsed time in microseconds by 58 and when to the value in feet for distance it is measured by dividing the elapsed time in microseconds by 148 . The distance calculated is displayed on to the LCD attached to sensor via the controller.

\section{APPLICATION AREA}

Ultra Sonic ranging has a wide area of applications. Ultra Sonic ranging is used in robotics applications [1][2] [3] [4] [5]. Measure the amount of liquid in a tank, Humidifiers, Sonar, Ultrasonography, burglar and testing [6] 


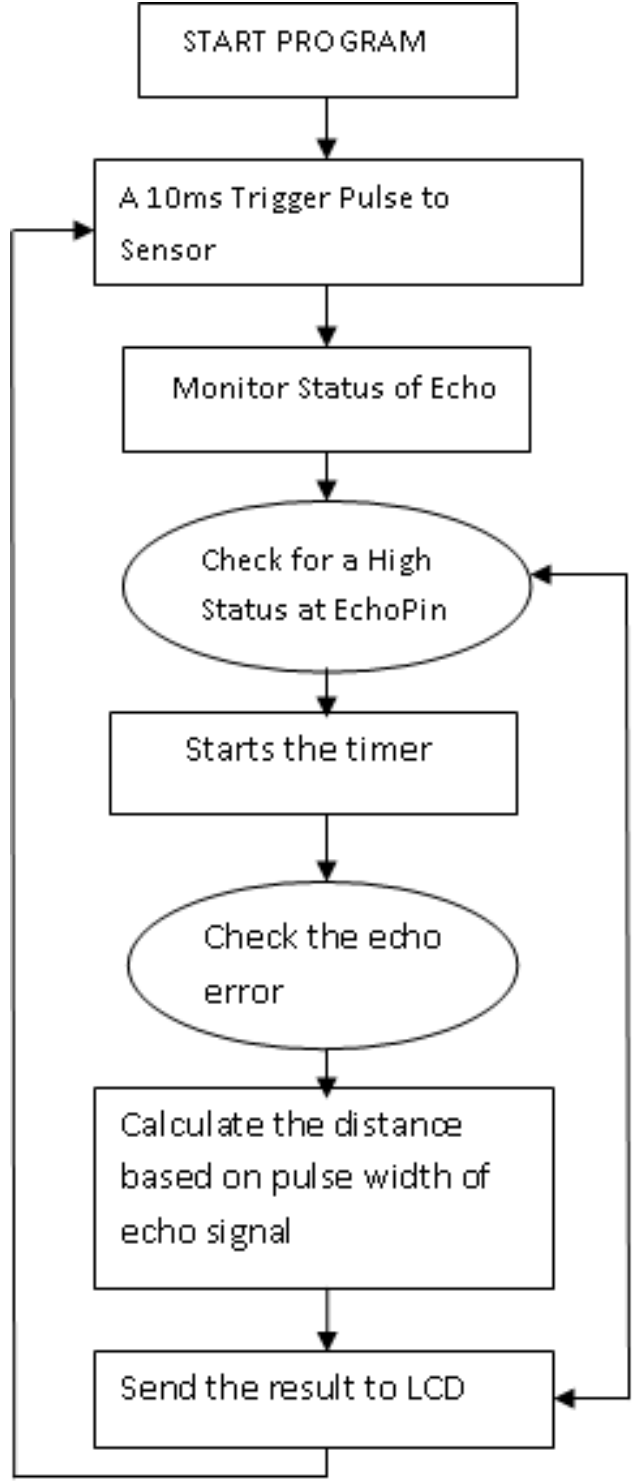

Fig 2

\section{ADVANTAGES}

UltraSonic range finders are of low cost. They are high speed for measurement which eventually reduces the time and increases the performance. Simple structures of Ultrasonic range finders are easy to manufacture. They work in severe weather therefore reliable.

\section{DISADVANTAGES}

Sharp sensors maximum range limited to $80 \mathrm{~cm}$. Ordinary sensors maximum range limited to $30 \mathrm{~cm}$. Poor resolution of wave beam [4]. Unexpected reflection phenomenon mirror [4].

\section{RESULT}

Result of this paper is that with the use of Ultra Sonic range finder the distance is measured with accuracy without creating hindrances in the sensitive areas like coal mines, water bodies, human bodies.

\section{CONCLUSION AND FUTURE SCOPE}

This paper discuss about how the problem of measuring distances in sensitive areas can be overcome by using Ultra Sonic sensors with the help of its algorithm the accurate measurement can be taken and displayed.

\section{ACKNOWLEDGEMENTS}

I would like to thank our University and laboratory faculty without them the completion of this project and research would not be possible.

\section{REFERENCES}

[1]. J. L. Crowley, "Dynamic world modeling for an intelligent mobile robot," in IEEE 7th Int. Conf. Pattern Recognition Proc., 1984, Montreal, PQ, Canada, pp. 207210.

[2]. "Navigation for an intelligent mobile robot," CarnegieMellon University, The Robotics Institute, Pittsburgh, PA, Tech. Rep., Aug, 1984.

[3]. C. Helmers "Ein heldenlesen," Robotics Age, pp. 7-16 and pp. 44-45, Mar/Apr. 1

[4]. H.P Moravec and A.Elfes, "High resolution maps from wide angle sonar," presented at the IEEE conf. Robotics and Automation, 1985.

[5]. C.Quick, "Animate versus inanimate," Robotics Age, pp. 15-17 Aug. 1984.

[6]. Design of an Ultra Sonic Distance meter, International Journal of Scientific and Engineering Research Volume 4, Issue 3, Mar. 2013,ISSN2229-5518.

[7]. Ciarcia, "Home in the range, An ultrasonic ranging system,"BYTE, Nov. 1980.

[8]. R. A. Cooke, "Microcomputer control of free ranging robots," in Proc. 13th Int. Symp, Industrial Robots and Robots, Chicago, IL, Apr.1983,pp.13.109-13.120.

[9]. H.R.Everett, "A second generation autonomous sentry robot, "Robotics Age, pp. 29-32, Apr.1985".

[10]. A multi element ultrasonic ranging array," RoboticsAge,pp.13-20,July1985.

[11]. J. P. Hermann et al., "Pattern recognition in the factory: An example," in Proc. 12th Int. Symp, Industrial Robots, Paris, France, 1982, pp.271-280.

[12]. G. Hoffstatter "Using the Polaroid ultrasonic ranging system."Robotics Age, pp. 35-37”, Sept. 1984.

[13]. J.Iijima,S. Yuta and Y .Kanayama, "Elementary functions of a self contained robot 'YAMABICO 3.1'," in Proc. Ilth Int. Symp. Industrial Robots, Tokyo, Japan, 1983, pp. 211-218.

[14]. D. L. Jaffe, "Polaroid ultrasonic ranging sensors in robotic applica-tions," Robotic Age, pp.23-30,Mar.1985

[15]. C. Jorgensen, W. Hamel, and C. Weisbin, "Autonomous robot navigation," BYTE, pp. 223-235, Jan. 1986.

[16]. H.Kuttruff, Room Acoustics, 2nd ed. London: Applied Science Publishers, 1979, pp. 77-80.

[17]. D. Lampe, "Robot sentries," Popular Sci., p. 20,Aug.1985.

[18]. L.Leifer, "Rehabilitative robotics, The Stanford robotic aid", presented at the Robotics WestConf,Sept.1981. 
[19]. "Rehabilitative robots," Robotics Age, pp. 4-14, May1981.

[20]. P. W. K. Lau, Robotic steering, "Internal Project of the Trent Polytechnic, Nottingham, England, 1981.

[21]. T. Lozano-Perez, "Automatic planning of manipulator transfer movements," IEEE Trans. Syst., Man., Cybern., vol. SMC-II, pp. 681-698, Oct.1981.

[22]. G. D. Maslin, "A simple ultrasonic ranging system," presented at the102nd Convention of the Audio Engineering Society, Cincinnati, OH, May12,1983 (reprinted in POLAROID Ultrasonic Ranging System Handbook, Application Notes/Technical Papers, supplied with sensor hardware kit).

[23]. J P. M. Morse, Vibration and Sound, 2nd ed. New York: McGrawHill, 1948, pp. 326-329.

[24]. H. F. Olson, Acoustical Engineering, 3rd ed. Princeton, NJ: VanNostrand, 1957, pp. 20-23.

[25]. Ultrasonic Ranging System, Polaroid Corporation, 1982. 